\section{Advanced prostate cancer can be predicted by PSA level measured up to 25 years earlier}

Lilja and colleagues previously reported that serum PSA level in men aged $\leq 50$ years was a strong predictor of prostate cancer diagnosed up to 25 years afterwards. However, the researchers concede that many prostate cancers do not cause morbidity or mortality and have, therefore, reanalyzed their data using locally advanced or metastatic disease at diagnosis as the clinical end point.

Participants for the case-control study were selected from a population-based cohort of 21,277 men from Sweden who provided blood samples between 1974 and 1986 when aged $\leq 50$ years. Cases of locally advanced or metastatic prostate cancer through 1999 were identified ( $n=161$; median 17 years from blood-sample collection to diagnosis) and controls were matched for blood-sample collection date and age (cases were matched with 3 (73\%), 2 (24\%) or 1 (3\%) controls). Researchers measured serum levels of total PSA, free PSA and human kallikrein 2 in the original blood samples.

Total PSA was strongly predictive of a subsequent diagnosis of advanced prostate cancer (area under the curve 0.791); the other two markers were much less strongly predictive. Even moderately raised serum PSA levels considerably increased the risk of advanced disease, and the $20 \%$ of the population with the highest PSA levels $(\geq 0.9 \mathrm{ng} / \mathrm{ml})$ accounted for $66 \%$ of the advanced cancer cases.

The researchers recommend that a PSA test before 50 years of age could select at-risk patients for intensive screening, which would optimize the benefit of screening whilst minimizing the potential for harmful overtreatment.

Original article Ulmert D et al. (2008) Prostate-specific antigen at or before age 50 as a predictor of advanced prostate cancer diagnosed up to 25 years later: a case-control study. BMC Med 6: 6

\section{Validation of the Partin Tables for staging prostate cancer in African American men}

The Partin Tables, nomograms for staging prostate cancer in patients undergoing radical prostatectomy, were developed and validated in a predominantly white cohort of men. In the US, the disease characteristics of African American men who present with prostate cancer differ from those of white patients. Heath et al. have compared the accuracy of the 2001 updated Partin Tables for predicting pathologic stage in African American and white patients.

The researchers pooled data from four multiethnic cohorts of men who underwent radical prostatectomy in the US, including a total of 3,748 patients (1,188 [32\%] African American, 2,560 [68\%] white). The accuracy of the Partin Tables was assessed by estimating areas under the receiver operating characteristic curve (AUC) for each ethnic group. Accuracy did not differ significantly between African American and white men for predicting organ-confined disease (AUC 0.73 vs $0.72 ; P=0.56$ ), extracapsular extension (AUC 0.62 vs $0.62 ; P=0.99$ ) or seminal-vesicle invasion ( 0.77 vs $0.79 ; P=0.53$ ). The Partin Tables predicted lymph node metastasis more accurately in African American men than in white men (AUC 0.76 vs $0.65 ; P=0.05$ ).

The authors conclude that the Partin Tables perform equally well for staging prostate cancer in African American and white populations. African American patients tend to present with higher PSA levels, higher disease grade and more-advanced disease; however, once the clinical characteristics of disease are known, ethnicity is not predictive of prognosis.

\section{Original article Heath EI et al. (2008) The effect of race/ ethnicity on the accuracy of the 2001 Partin Tables for predicting pathologic stage of localized prostate cancer. Urology 71: 151-155 \\ Risk of prostate cancer is not affected by prediagnosis sex-hormone levels}

Studies that examine the effects of circulating endogenous sex hormones on the risk of prostate cancer have yielded mixed results: some indicate modest associations, but most are insufficiently powered to supply robust conclusions. The Endogenous Hormones and Prostate Cancer Collaborative Group performed a pooled analysis of 18 prospective studies, with the aim of uniformly assessing the association between serum levels of various sex hormones and risk of prostate cancer.

The analysis included data from 18 prospective studies, in which all patients had 